\title{
Management of Heterotopic Ossification of the Elbow in Patients with Elbow and Brain Injury a Retrospective Study
}

\author{
D Skouteris ${ }^{1}$, P Tsiasiotis ${ }^{1}$, G Tsikouris ${ }^{2}$ and V Psychoyios ${ }^{1 *}$ \\ ${ }^{1}$ Hand \& Upper Extremity Service, Asclepeion Hospital, Greece \\ ${ }^{2}$ Orthopaedics \& Sports Medicine Centre, Greece
}

*Corresponding author: Vassilios Psychoyios, Department of Orthopaedic, Hand Service, Asklepieion Hospital, Athens, Greece, Vasileos Paulou 1 Str. 16673, Voula, Athens, Greece

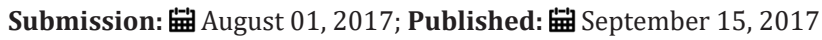

\begin{abstract}
The elbow is a highly constrained hinge joint with a high propensity for stiffness after bony or soft tissue injury, and heterotopic ossification (HO) after brain injury is considered one of the most debilitating causes resulting in severe limitation of motion and everyday activities.

The purpose of the present retrospective study was to report the results of surgical treatment for post-traumatic elbow stiffness caused by HO in patients with elbow trauma and brain injury necessitating admission to intensive care unit.

Although surgical treatment of $\mathrm{HO}$ is technically demanding and accompanied by high complication rates, reasonable outcome can be expected regardless of preoperative ROM or the type of brain injury, provided that upper limb does not a have any neurological deficit. While some loss of motion from what significantly gained intra-operatively can be expected, a majority of patients who actively participate in their post-operative physical therapy program will achieve an optimal outcome by preserving a functional arc of motion.
\end{abstract}

Keywords: Heterotopic ossification; Elbow joint stiffness; Brain injury

Abbreviations: HO: Heterotopic ossification; ICU: Intensive Care Unit

\section{Introduction}

The particular anatomical features of the elbow joint and its relation to the hand and shoulder lead and define the functional deficits after bony or soft tissue injury. Heterotopic ossification is considered one of the most debilitating causes, which almost always results in severe limitation of motion or complete ankylosis.

The prevalence of clinically significant $\mathrm{HO}$ in the traumatic brain injury population is estimated to be $10-20 \%$ [1] or even higher according to the literature [2]. Significant risk factors for HO development include trauma, burns, immobility, limb spasticity, and prolonged coma [3-5]. In traumatic brain injury HO tends to occur in the connective tissue between the muscle planes and not within the muscle itself [6], affecting in decreasing frequency the hips, elbows, shoulders, knees, and rarely, the thigh [1].

Recent studies reported more favorable surgical results in patients with HO than in those with other causes of stiffness [7-9]. However, HO has been considered as a poor prognostic factor after surgical release with respect to recovery of elbow motion, with some of the studies reporting a recurrence rate of $10 \%$ to $24 \%$ or results showing fair to poor outcomes in more than $30 \%$ of studied patients after surgical treatment [10]. The majority of the factors that lead to these poor outcomes still remain poorly understood $[10,11]$.

The purpose of the present retrospective study was to report the results of surgical treatment for post-traumatic elbow stiffness caused by $\mathrm{HO}$ in patients with elbow trauma and brain injury necessitating admission to intensive care unit, treated by the senior surgeons (G.T) and (V.P).

\section{Patients and Methods}

Between September 2000 and October 2012, 28 patients who fulfilled the following criteria were treated with open surgical release by one of the two senior surgeons (G.T) or (V.P.): (1) Posttraumatic stiff elbow (2) Traumatic brain injury with admission to ICU unit (3)total arc of motion of less than $100^{\circ}$ (4) HO visible on preoperative X-ray (5) skeletally mature elbows without a history of trauma and limitation in range of motion (ROM) before injury, (6) evidence of union and stability of the joint, and (7) a normal or minimally involved ulnohumeral joint (Figure 1). 


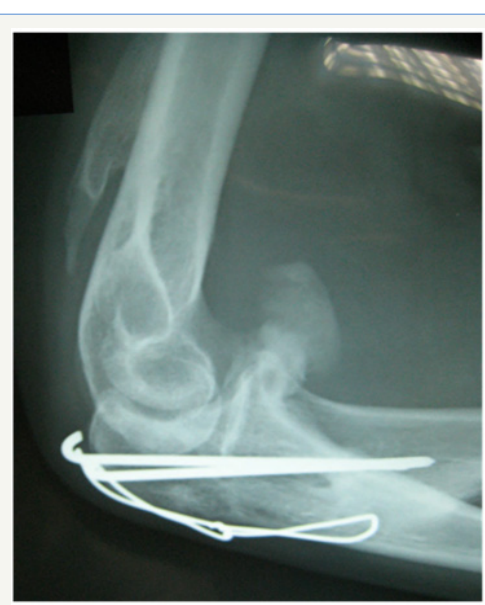

Figure 1: Typical case of elbow $\mathrm{HO}$ after olecranon fracture and brain injury. This patient sustained a subarachnoid hemorrhage and admitted to ICU for 20 days.

We were able to contact and re-examine 24 patients. There were 16 men and 8 women with a mean age of 37 years (range 18-

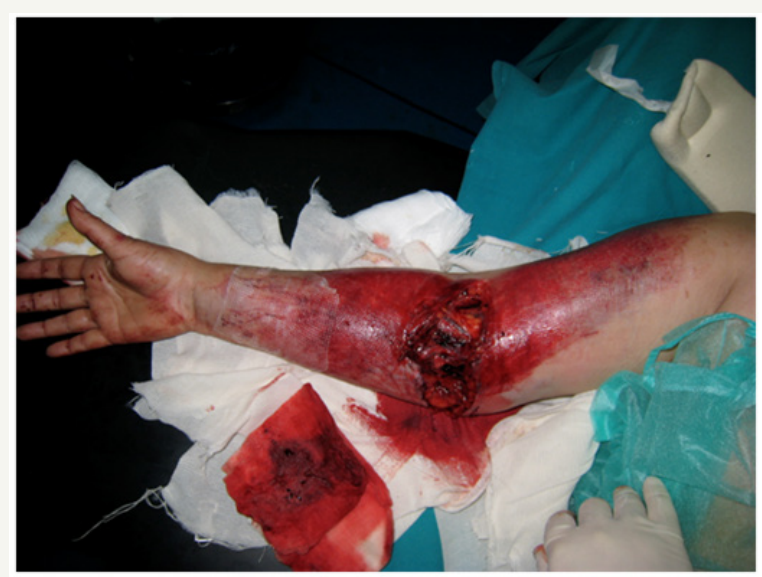

67). In seventeen patients the dominant arm was involved. Two patients had been lost from the follow up but their medical records from the last outpatient clinic visit were available. One patient died of unrelated reason and one patient refused to be re-examined. We included in this retrospective study 24 patients that we were able to re-examine clinically and radiographically. The mean follow-up was 93.2 months (range, 44-201 months).

Regarding the head injury eight patients sustained a subdural hematoma, five an epidural hematoma, four a subarachnoid hemorrhage, and seven a diffuse cerebral edema (Table 1). Six patients underwent a neurosurgical procedure on average of approximately five hours after admission to the hospital. The elbow trauma consisted of distal humerus fracture in five patients, an olecranon fracture in three, in complex fracture dislocation of the elbow in nine, radial head fracture in three, an elbow dislocation in two, an MCL rupture in one and an open elbow dislocation in one patient (Table 2) (Figure 2). Twenty patients underwent operative fixation of their lesions. The average duration to ICU was 29 days (range 17-86 days).

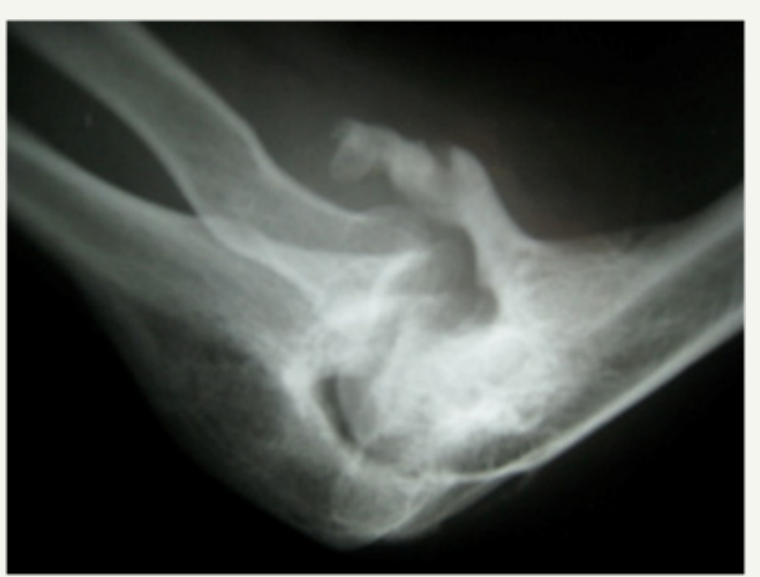

Figure 2: a) Open elbow dislocation in 27 yrs old patient who suffered from traumatic epidural hematoma.

b) Almost complete elbow ankylosis after 22 months of the original injury.

Table 1: Head injury lesions.

\begin{tabular}{|c|c|}
\hline Subdural hematoma & 8 \\
\hline Epidural hematoma & 5 \\
\hline Subarachnoid hemorrhage & 4 \\
\hline Cerebral edema & 7 \\
\hline
\end{tabular}

Table 2: Elbow injuries.

\begin{tabular}{|c|c|}
\hline Distal humerus fracture & 5 \\
\hline Olecranon fracture & 3 \\
\hline Radial head fracture & 3 \\
\hline Complex elbow fracture dislocation & 9 Total \\
\hline Terrible triad & 3 \\
\hline Posterior Monteggia lesion & 2 \\
\hline Transolecranon fracture dilocation & 1 \\
\hline MCL rupture & 1 \\
\hline Open elbow dislocation & 2 \\
\hline
\end{tabular}




\section{Surgical management}

Excision of the heterotopic bone (formed) as well as elbow soft tissue contracture release was performed utilizing well-described surgical techniques in the literature [8,12-14]. Persistent loss of elbow motion causing disability, limitation of daily activities, pain and duration of the symptoms for at least nine months with no sign of improvement despite physical therapy were the clinical indications for surgical intervention. A negative bone scan and normal levels of alkaline phosphatase were the main laboratory indicators of heterotopic bone maturity until 2004. Since then we abandoned their use, as according to our limited experience and the literature in matter, ALP is not considered a reliable screening method for early HO detection $[15,16]$. Furthermore, bone scintigraphy has limited value for evaluating the maturity of ossification and is little used in clinical practice [8]. Our main criteria for excision after 2004 were the clinical status, as described above, and a stabilized lesion radiographically.

Approach was determined by previous skin incisions, presence of ulnar neuropathy, location of the heterotopic bone and status of the radial head. A long posterior midline incision was used in 18 patients, a combination of medial and lateral incision in two, a lateral in two, amedial in one and an anterior in one (Figure 3).
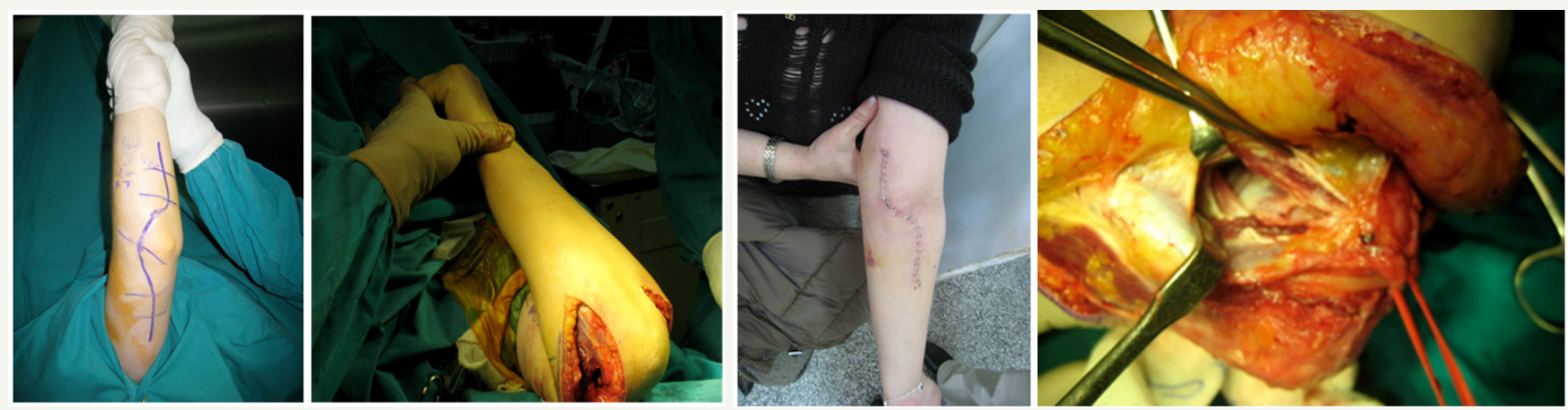

Figure 3: Various approaches utilized for the HO excision from elbow joint

a) A long posterior incision gives access posteriorly, anteriorly medially and laterally to the elbow joint.

b) Medial and lateral approach utilized separately to excise HO from the medial lateral and lateral aspect of the elbow joint.

c) An anterior approach to the elbow joint. Utilized very rarely and in specific cases of HO location at the anterior elbow surface.

d) A medial approach to elbow joint. In this particular case after excision of the HO through the flexor-pronator mass.

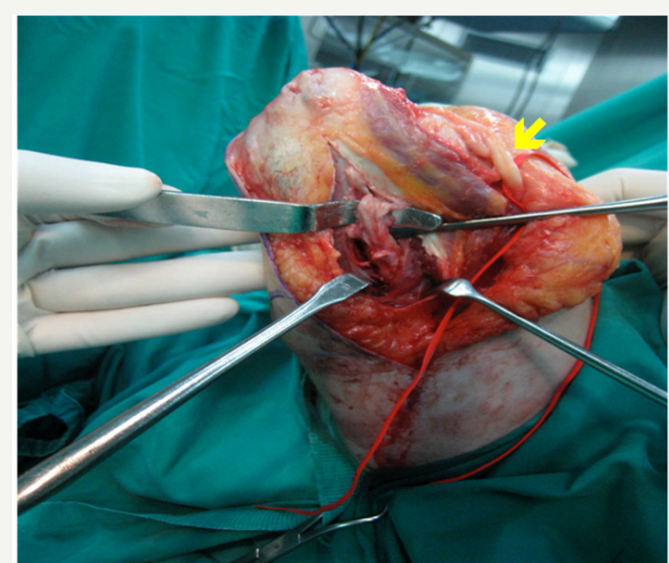

Figure 4: Large medial and lateral fasciocutaneous flaps. Identification and protection of the ulnar nerve (yellow arrow).

In a posterior approach a large medial and lateral full thickness fasciocutaneous flaps were created and the ulnar nerve was identified and released up to the proximal aspect of the flexor carpi ulnaris (FCU) muscle and protected (Figure 4). After dissection of the triceps muscle from the posterior humerus, a complete excision of the posterior capsule was performed as well as excision of heterotopic bone from the posterior aspect of the joint, especially around the olecranon and the olecranon fossa. If necessary, the posterior band of the MCL was released, until gaining flexion up to 130 degrees or more. When forearm motion was limited the
Kocher interval was developed and an anterior capsulectomy was performed in combination with excision of heterotopic bone whenever it was present. In five cases a radial head excision was undertaken due to enormous enlargement from chondral metaplasia, after radial head fracture (Figure 5). In six cases a medial approach through the flexor-pronator mass was developed, one primarily and the rest as a part of another approach, in order to completely excise the anterior capsule and achieve full extension of the joint.
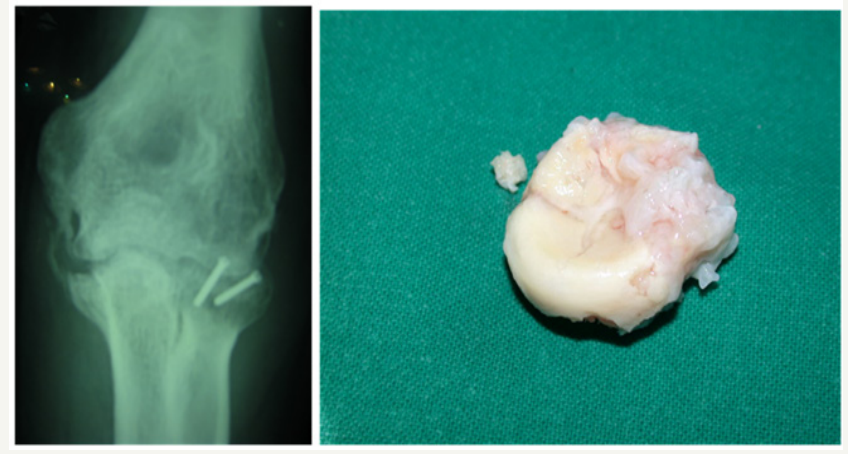

Figure 5: Elbow HO after brain injury and Radial head fracture.

a) HO in the lateral and medial side.

b) Radial head chondral metaplasia

Hardware for fracture fixation was removed in all patients. In one patient a supracondylar fracture was created attempting to gain full extension, and treated with k-wires (Figure 6). In three 
patients a pie crust triceps lengthening/release was performed to gain additional flexion.
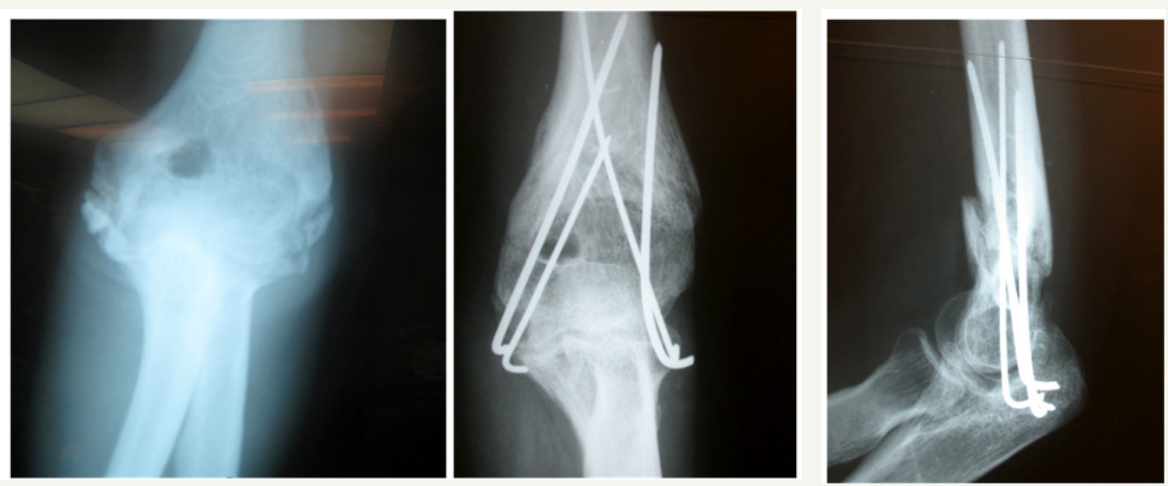

Figure 6: Iatrogenic fracture after excision of the Elbow HO in attempting to gain full ROM.

a) Elbow $\mathrm{HO}$ affecting all the elbow surfaces after elbow dislocation.

b) Supracondylar elbow fracture after $\mathrm{HO}$ excision, treated with k-wires.

c) Profile view from the same elbow xray.

The lateral collateral ligament was accidentally detached from its humeral insertion in one patient and repaired with transosseous sutures. The ulnar nerve was transposed subcutaneously in twentyone patients either as a preventive or as a therapeutic procedure. Bone wax was applied onto all bony surfaces from which bone was excised.

A long arm splint with the elbow in full extension was applied onto the anterior aspect of the joint (Figure 7). Active assisted and passive flexion-extension exercise begun the first postoperative day. Indomethacin $75 \mathrm{mg}$ was administered twice daily for six weeks in order to prevent a new ectopic bone formation. An intensive physiotherapy program was followed and a turnbuckle splint was applied after edema subsidence, usually after the third postoperative week. The mean duration of physical therapy was 5 months and the mean duration of the turnbuckle splint usage was 11 months. No adjuvant radiation therapy was used, although its true value in HO management is still debated. According to Cipriano et al. [17] local radiation was not effective in patients with neurogenic HO. On the other hand, studies on patients with HO after spinal cord injuries treated with adjuvant radiation therapy indicate significant improvement in ROM $[18,19]$.

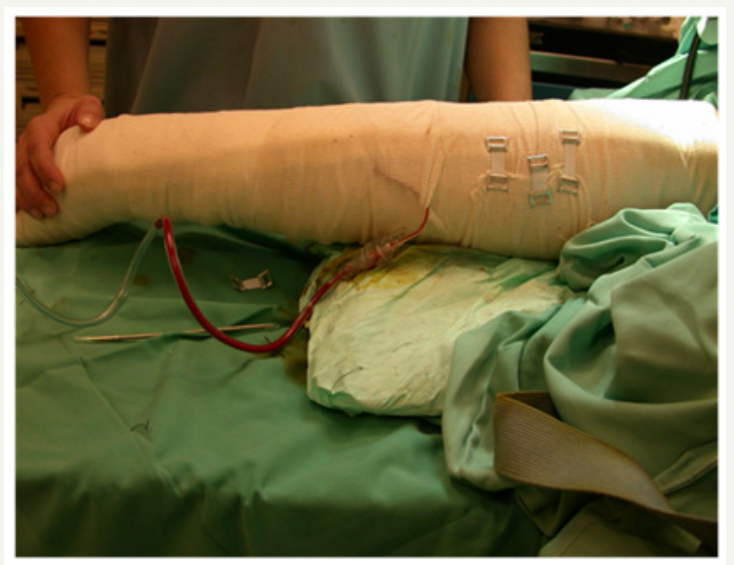

Figure 7: A long arm anterior splint applied with elbow joint in full extension.

\section{Results}

The average arc of elbow motion improved from $40^{\circ}$ (range $0^{\circ}$ $80^{\circ}$ ) pre operatively to $110^{\circ}$ (range $0^{\circ}-140^{\circ}$ ), with an improvement of $70^{\circ}$ at the final follow-up evaluation. The mean Mayo Elbow Performance Index MEPI score was 89.9 (range, 60-100). Results were good to excellent in 18 patients, fair in 5 patients, and poorin one (Table 3). Among the 24 study patients, 19 (79.1\%) obtained a total ROM of 100 oat the final evaluation, and 3 underwent repeated surgical release due to recurrence of contracture. In two of them a recurrence of HO was present 9 months on average postoperatively. Patients who required repeated surgical release had an average arc of flexion-extension of $70^{\circ}$ at the time of their second surgery and an average of $90^{\circ}$ after their second elbow release at final evaluation for the needs of the present paper.

Table 3: Preop and Postop ROM and the Mayo Elbow Performance Index.

\begin{tabular}{|c|c|c|}
\hline & Preop & Postop \\
\hline Av E/F & $45(35-85)$ & $110(0-140)$ \\
\hline Av S/P & $100(45-55)$ & $165(75-85)$ \\
\hline MEPI score & 45 & 89.1 \\
\hline
\end{tabular}

Five patients did not gain an arc of $>100$, and in two of them recurrence of $\mathrm{HO}$ was regarded as the major reason of failure. In the rest three patients a severe osteoarthritis was developed and considered as the main reason of the failure.

There was one iatrogenic injury to the lateral ligamentous complex and one iatrogenic fracture of the distal humerus, treated accordingly. Three patients developed a mildly valgus deformed elbow without clinical consequences. Three patients underwent a second procedure, a transmuscular ulnar nerve transposition together with internal neurolysis, due to tardy ulnar nerve palsy 75 months on average of 100 months after the index procedure. Recurrent $\mathrm{HO}$ was observed in two patients postoperatively, one of them denied any further treatment. 


\section{Discussion}

The goal of our study was to assess the outcome in improvement of the range of motion of the elbow and of the daily activities after surgical release of elbow stiffness caused by HO after elbow trauma and concomitant brain injury.

Thus we can state that a very reasonable outcome can be expected regardless of preoperative ROM or the type of brain injury, provided that upper limb does not a have any neurological deficit.=

In the past $\mathrm{HO}$ has been considered as a poor prognostic factor after elbow trauma $[10,20,21]$. Due to the extensive loss in ROM, the high recurrence rates of $\mathrm{HO}$ and complications after surgical treatment, the decision making for operative intervention was a less attractive option.

Nowadays, although surgical treatment of HO is technically demanding and accompanied by high complication rates, there are studies which report good results after surgical treatment of elbow stiffness due to HO [4,22-24]. Accordingly, there are studies that demonstrate better results for the HO group in comparison with the capsular contracture group after surgical treatment 7. However, the are no studies evaluating the outcome of surgical release in patients with elbow trauma and brain injury, admitted for a period of time to ICU.

We attempted to evaluate the results especially in this subcategory of patients and to extract conclusions, although retrospectively, regarding the feasibility of the procedure, the outcome regarding ROM and ability to perform daily activities. Furthermore due to complexity of those procedures we encountered the potentials risks and tried to find ways to avoid or fix them.

Excision of heterotopic bone as well as release of contracture or ankylosis about the elbow requires a very sound knowledge of anatomy in this particular area. A posterior skin incision offers a global approach to elbow surfaces and deep structures. Substantial improvement in elbow and forearm range of motion can be anticipated following the procedure described although a high complication rate early or delayed can interrelate these procedures. Every patient should be treated with an aggressive rehabilitation protocol that includes prolonged physical therapy as well as use of turnbuckle splint14. Adjuvant treatment with indomethacin should be utilized in every patient as various studies seem to support the efficacy of NSAIDs and indomethacin in particular [4,25]. While some loss of motion from what significantly gained intra-operatively can be expected, a majority of patients who actively participate in their physical therapy program will achieve an optimal outcome by preserving a functional arc of motion.

\section{Conflict of Interest}

All the authors certify that they have NO affiliations with or involvement in any organization or entity with any financial interest (such as honoraria; educational grants; participation in speakers' bureaus; membership, employment, consultancies, stock ownership, or other equity interest; and expert testimony or patent- licensing arrangements), or non-financial interest (such as personal or professional relationships, affiliations, knowledge or beliefs) in the subject matter or materials discussed in this manuscript.

\section{References}

1. Chan, Kwai-Tung (2005) Heterotopic Ossification in Traumatic Brain Injury. American Journal of Physical Medicine \& Rehabilitation 84(2):145-146.

2. Cipriano CA, Pill SG, Keenan MA (2009) Heterotopic ossification following traumatic brain injury and spinal cord injury. J Am Acad Orthop Surg 17(11): 689-697.

3. Abrams GD, Bellino MJ, Cheung EV (2012) Risk factors for development of heterotopic ossification of the elbow after fracture fixation. J Shoulder Elbow Surg 21(11): 1550-1554.

4. Sandeep KN, Suresh G, Gopisankar B, Abhishek N, Sujiv A (2017) Does excision of heterotopic ossification of the elbow result in satisfactory patient-rated outcomes? Malays Orthop J 11(1): 35-40.

5. Garland DE, Orwin JF (1989) Resection of heterotopic ossification in patients with spinal cord injuries. Clin Orthop Relat Res 242: 169-176.

6. Jensen LL, Halar E, Little J, Brooke MM (1987) Neurogenic heterotopic ossification. Am J Phys Med Rehabil; 66(5): 351-363.

7. Lindenhovius AL, Linzel DS, Doornberg JN, Ring DC, Jupiter JB (2007) Comparison of elbow contracture release in elbows with and without heterotopic ossification restricting motion. J Shoulder Elbow Surg 16(5): 621-625.

8. Filho GM, Galvao MV (2010) Post-traumatic stiffness of the elbow. Rev Bras Ortop 45(4): 347-356.

9. Ring D, Jupiter JB (2004) Operative release of ankylosis of the elbow due to heterotopic ossification. Surgical technique. J Bone Joint Surg Am 86(Suppl 1): 2-10.

10. Koh KH, Lim TK, Lee HI, Park MJ (2013) Surgical treatment of elbow stiffness caused by post-traumatic heterotopic ossification. J Shoulder Elbow Surg 22(8): 1128-1134.

11. Moreta J, Luis Martinez-de los Mozos J (2014) Heterotopic ossification after traumatic brain injury, Chapter 15. Traumatic Brain Injury 12221228.

12. Morrey BF (1990) Post-traumatic contracture of the elbow. Operative treatment, including distraction arthroplasty. J Bone Joint Surg Am 72(4): 601-618.

13. Morrey BF (2005) The posttraumatic stiff elbow. Clin Orthop Relat Res 431: 26-35.

14. Dodds SD, Hanel DP (2006) Heterotopic Ossification of the Elbow. In: Trumble TE, (Ed.), Wrist and Elbow Reconstruction \& Arthroscopy. Rosemont, American Society for Surgery of the Hand, USA, pp. 425-438.

15. Singh RS, Craig MC, Katholi CR, Jackson AB, Mountz JM (2003) The predictive value of creatine phosphokinase and alkaline phosphatase in identification of heterotopic ossification in patients after spinal cord injury. Arch Phys Med Rehabil 84(11): 1584-1588

16. Citak M, Grasmucke D, Suero EM, Cruciger O, MeindL R, et al. (2016) The roles of serum alkaline and bone alikaline phosphatase levels in predicting heterotopic ossification following spinal cord injury. Spinal Cord 54(5): 368-370.

17. Cipriano C, Pill SG, Rosenstock J, Keenan MA (2009) Radiation therapy for preventing recurrence of neurogenic heterotopic ossification. Orthopedics 32(9).

18. Sautter-Bihl ML, Hultenschmidt B, Liebermeister E, Nanassy A (2001) Fractionated and single dose radiotherapy for heterotopic bone formation in patients with spinal cord injury. A phase I/II study. Strahlenther Onkol 177(4): 200-205. 
19. Sakellariou VI, Grigoriou E, Mavrogenis AF, Soucacos PN, Papagelopoulos PJ (2012) Heterotopic Ossification Following Traumatic Brain Injury and Spinal Cord Injury: Insight Into the Etiology and Pathophysiology. J Musculoskelet Neuronal Interact 12(4): 230-240.

20. Jupiter JB, O'Driscoll SW, Cohen MS (2003) The assessment and management of the stiff elbow. Instr Course Lect 52: 93-111.

21. Viola RW, Hastings H (2000) Treatment of ectopic ossification about the elbow. Clin Orthop Relat Res 370: 65-86.

22. Salazar D, Golz A, Israel H, Marra G (2014) Heterotopic ossification of the elbow treated with surgical resection: risk factors, bony ankylosis, and complications. Clin Orthop Relat Res 472(7): 2269-2275.
23. Baldwin K, Hosalkar HS, Donegan DJ, Rendon N, Ramsey M, et al. (2011) Surgical resection of heterotopic bone about the elbow: an institutional experience with traumatic and neurologic etiologies. J Hand Surg Am 36(5): 798-803.

24.Park MJ, Chang MJ, Lee YB, Kang HJ (2010) Surgical release for posttraumatic loss of elbow flexion. J Bone Joint Surg Am 92(16): 26922699.

25.Fransen M, Neal B (2004) Non-steroidal anti-inflammatory drugs for preventing heterotopic bone formation after hip arthroplasty. Cochrane Database of Syst Rev 3: CD001160. 